\title{
Reflections of Parents and Parent Work Practitioners on the Changing Experiences and Circumstances of Parenting in Hong Kong
}

\author{
Siu-Ming To, Ching-Man Lam, and Yuk-Yan So
}

\begin{abstract}
Whereas previous research has highlighted the importance of understanding how parenting has become a risky venture from the perspective of risk society, few attempts have been made to explore how parents and practitioners make sense of the changing experiences and circumstances of parenting. By reporting the findings of a focus group study on 43 Hong Kong parents' and 33 parent work practitioners' reflections on the changing parental experiences over the past decades, as well as the changing circumstances in which parents raise children, this article discusses how both parents and practitioners found considerable intergenerational changes in parenting and described parenting in today's Hong Kong society as a risky venture fraught with boundless duties and challenges. The findings suggest that on the one hand, parents' self-perception is shaped by the risk discourses; on the other hand, parents who are able to reconstitute their reflexive selves can create a space, no matter how tenuous, within which they can have a more positive view of themselves and their children.
\end{abstract}

Index Terms-Parenting, parenthood, risk society, social work.

\section{INTRODUCTION}

Recent research has drawn our attention to the interplay of a number of historical, social, economic, as well as cultural factors that have drastically changed the social and cultural expectations for parenting and brought about the development of parenting phenomenon such as intensive parenting [1] and anxious parenting [2]. Such factors include the transformation of families in the aftermath of industrialization and modernization [2], the emergence of a number of discourses on parenting and childrearing [1], and the precariousness and disorientation of life in late modernity [3] By reporting the findings of a focus group study on Hong Kong parents' and parent work practitioners' reflections on the changing parental experiences over the past decades, as well as the changing circumstances in which parents raise children, this article discusses how the heightened sense of risk consciousness, hand in hand with an increased emphasis on parental efforts to manage the life risks encountered by children, help reconstruct the meaning of parenting in the Hong Kong Chinese context.

Scholars like Beck [4] and Giddens [5] have pointed out that while modernity has rendered certain aspects of lives safer than previous eras, it has paradoxically introduced and

Manuscript received March, 1 2019; revised June 2, 2019. This study was financially supported by the General Research Fund 2014/15 of the Research Grant Council, The Hong Kong SAR Government (Ref No. U414053)

The authors are with the Chinese University of Hong Kong, HK (e-mail: siumingto@cuhk.edu.hk, yyso@swk.cuhk.edu.hk). even manufactured new risks and uncertainties that are largely unknown and unimaginable to previous eras [5]. Risks and uncertainties have hence become key features of contemporary societies. Individuals are constantly engaging in assessing and managing risks in all areas of their lives. These risks and uncertainties in late modernity are further channelled into the arena of parenting [3], which drive parents to protect their children from various forms of risks in the world [6]. Furthermore, the discourse of globalization urges parents to accept that a competitive life is essential for their children to survive in the globalized society. Nelson [6] has argued that as long as extreme income inequality prevails in the globalized world, which has made the consequences of failure too severe for the coming generation to bear, parents are not likely to change their style of anxious parenting, lest their children would lose the edge and fail to survive in this highly competitive globalized society.

Following rapid changes in the cultural, social, and economic contexts, parenting in Hong Kong has undergone significant changes over the past few decades. Adopting the perspective of Beck on risk society [4], Chan [7] further argued that the trend of modernization has led to the dismantling of old family norms and systems and the establishment of new ones, resulting in a greater sense of choices in determining one's life courses well as the arrangements for families. As such, family structures and values in Hong Kong have become more uncertain, diverse, flexible, and even risky [7]. For instance, in face of the economic downturn, the escalating cost of childrearing, and a highly competitive education system for children, young couples tend to postpone their first childbirth, bear fewer children or choose to be childless [8]. The image of the precious child hence prevails, and new social norms and cultural expectations for meeting the developmental needs of a child and providing intellectually enriching activities thus emerged, in addition to the traditional expectations of providing food and shelter [9]. Together with the erosion of intergenerational support, the handling of the precious child has thus stirred up a lot of concerns and uncertainties of parents, resulting in the belief that childhood must be carefully crafted and managed so that the child can secure a prosperous future [8].

The landscape and cultural understanding of parenting have also undergone significant changes with the introduction of parent education as the core component of Family Life Education Service in Hong Kong some thirty years ago. Embedded within a human development framework transplanted from the West [10], most of the existing parent education programs are modeled on approaches developed in Western societies [8], [11]. 
Although past studies have shown that these programs are generally effective in many aspects, from reducing parental stress to improving parent-child relationships to tackling children behavioral problems [12], [13], premised on objectivist science and instrumental rationality, these programs have given rise to some unintended results. First, these programs have constructed the inadequate parent discourse which highlighted the needs of parents to learn new knowledge and skills of parenting from experts [8]. This deficit view of parents has negated parents' ability and knowledge and hence contributed to a negative self-image of parents, who always think they are lacking something, and hence actively seek expert advice and help [14], [15]. The notion that parents need to learn from experts in order to be good parents has become a widely held view of the public in Hong Kong. A discourse which highly respects the authority of experts to the negation of traditional values, knowledge, and practice hence developed.

\section{RESEARCH GAP AND RESEARCH OBJECTIVE}

Whereas the above review of literature has highlighted the importance of understanding how parenting has become a discourse object from the perspective of risk society, it has not touched on how parents and practitioners make sense of the changing experiences and circumstances of parenting. Moreover, risk society is a Western concept that should be subject to scrutiny regarding its applicability in understanding the beliefs and patterns of behaviors of Hong Kong parents, who may still hold the traditional Chinese values but at the same time living in Western ways [16]. In this regard, the current focus group study aimed to examine the changing experiences and circumstances of parenting of Chinese parents living in Hong Kong.

\section{METHOD}

\section{A. Research Design}

This focus group study is a part of a three-year research project ("Raising children in Hong Kong: A mixed-methods study of generational shifts in parental beliefs and practices") that employed a mixed-methods design including four interrelated stages: 1) an archival study of discourses on parenting; 2) a narrative inquiry of 60 people's experience in being parents across five decades through in-depth individual interviews; (3) a large scale quantitative survey on generational shifts in parental beliefs and practices over the last half century, and (4) focus group interviews with parents and parent work practitioners. At the last stage of this research, five parent inquiry groups made up of parents of different genders and cohorts, and five practitioner inquiry groups made up of social workers, teachers, parent work practitioners, and academic researchers on parenting were undertaken.

\section{B. Participants}

With regard to the parent inquiry groups, two groups were for older parents with their children born in the 1970's or before, 1980's, and 1990's. Three groups were for younger parents with their children born in the 2000's, and 2010's.
They were recruited through NGOs operating elderly services and a research institute studying aging $(n=10)$, universities $(n=8)$, a secondary school $(n=8)$, a primary school $(n=8)$, and a kindergarten $(n=9)$. A total of 43 participants were successfully recruited and interviewed in these five focus groups.

With regard to the practitioner inquiry groups, two groups were for social workers, two groups were for parent work practitioners and researchers, and one group was for teachers. They were recruited through local NGOs $(n=15)$, universities $(n=6)$, institutes/associations related to parent work $(n=6)$, and schools $(n=6)$. A total of 33 participants were successfully recruited and interviewed in these five focus groups. Among the participants, 5 were males and 28 were females. On average, the participants have been engaging in parent work for 13.15 years.

\section{Data Collection}

We obtained ethical approval from the Survey and Behavioral Ethics Committee of the Chinese University of Hong Kong. The focus groups were led by the investigators and trained interviewers who flexibly followed an interview schedule that contained grand-tour questions and suggested probes to scaffold participants' narrations. Each focus group lasted for 1.5 to 2 hours. All focus groups were conducted in Cantonese, a dialect in China. Questions were centered on the exploration of parents' personal accounts of the possible changes in parental values, beliefs, and practices over the past decades, their understanding of the contextual factors influencing the changes in parenting, their perception of the professional knowledge and expert advices of parenting, and their views on the development of parent education in Hong Kong. As a token of appreciation, all parent participants received a $\mathrm{HK} \$ 100$ supermarket coupon and all practitioners received a souvenir after the completion of the focus groups.

\section{Data Analysis}

The content of the focus groups were fully transcribed after the interviews. During the focus groups, two research assistants took reflective notes on themes and expressions having direct bearing on the research questions. A post-interview initial reading of the transcripts and reflective notes was conducted by a research assistant and an investigator not physically present in the interviews; this produced a triangulated written summary of the interviews. At the next stage, a research assistant initially coded the data and identified significant themes across the entire data set. Then, an investigator read the transcripts and verified or modified the codes and themes generated by the research assistant. After that, the investigators (the authors of this article) cross-checked the themes, made sense of the connections that were emerging between themes, and extracted quotations that best illustrated those themes.

\section{FINDINGS}

The following meaningful themes were identified from the narratives of the participants. While most of the participants did not use academic terms such as risk society and intensive parenting, they used language relevant to these subjects when they recounted their perceptions and experiences. These 
themes unravelled and summarized the reflections of the participants on the changing experiences and circumstances of parenting in Hong Kong.

\section{A. Intergenerational Changes in Parental Goals and Responsibility}

The narratives of the participants vividly illustrate the intergenerational changes in parental goals and responsibility. For older parents, they held that the main parental goal is to try their best to provide adequate tangible resources and education for their children so that they can earn a living, and their parental responsibilities can be regarded as fulfilled when their children have grown up. The following account of a father illustrates the typical perception of older parents:

"As for me, when I was young, there were 12 siblings in my family. That was why my father was forced to earn a living and seldom took care of us. We were taken care by mum... She also told me that children should be provided with education, or they would be useless when they have grown up. I have kept this statement [in my heart] till now. This statement is true. If he is capable to study, then I should let him. No matter how hard I have to work, I must finance him." (Older Generation, Group 2, Father A)

Meanwhile, the older parents witnessed the changes of parenting in their own generation and the next generation. They commented that compared to parenting in the old days, today's parents need to face the increasing demand of supporting their children extensively and unfailingly, particularly when their children cannot gratify their needs after they have grown up. An older mother said:

"To summarize, [in the past] the simplest request of a parent is that you [as a young adult] are able to support your own living. Actually this is very important. As you can see, the youngsters of this generation take home no money and even need parents to support them back. This is really pathetic... Sometimes I hear parents mention situations that their son doesn't take home money and they need to support him back." (Older Generation, Group 2, Mother A)

While revolving around the basic educational and developmental needs of children has been regarded as a parental responsibility in the older generations of parents, the younger generations of parents put more effort into nurturance and cultivation of potential for their children. They perceive childrearing and child nurturing as a life project warranting much parental input, and thus parents need to equip themselves with knowledge and skills of parenting in a more systematic way. The following two narratives best illustrate such a view:

"How did the generation of our parents help us build a foundation for development? That's actually simple. You need to be a good person, be someone with good personality... The concept of teaching you how to be a person hasn't changed... But now, for example we always complain that school hasn't taught enough. Some 'monster parents' put children in a kindergarten in the morning and another in the afternoon. How do they conceptualize this? They think the more the better, just fill the children up." (Younger Generation, Group 1, Father A)

"I guess the parents of the older generation might not have done nothing. They were not expressive in their execution, or they were not doing this in a very systematic or methodical way, not like how parents nowadays are doing, such as having lessons and group discussions, or reading books." (Younger Generation, Group 1, Mother A)

Echoing the voices of parent participants, both practitioners and teachers commented that instead of meeting their children's basic materialistic and educational needs, today's parents are more concerned about how to support them to maintain the economic status and secure their future opportunities in this highly competitive society. A social worker and a teacher have elaborated this point of view:

"Parents in the past were worried till [their children study] junior or senior secondary schools. As for now, parents would still come to seek help when their children are already in university. Some parents would even help queueing up to buy mortgage. Some parents would seek advice from social worker regarding if they should use their retirement saving to help children pay their first down payment." (Social Worker C)

"I think that's because of the change of our economic environment. The older generation had to keep up with basic needs in daily life. Hong Kong has become richer in material sense nowadays. What parents' consider now is how to give their children the best." (Teacher A)

\section{B. Perceived Changing Contexts that Affect the \\ Experiences and Circumstances of Parenting}

When asked about the factors affecting the changing experiences and circumstances of parenting, the younger generations of parents pointed out that Hong Kong families have undergone various significant changes over the past two decades. First, in face of a fluctuating economic environment and a continued blooming property market, parents have little choice but to provide financial support to their children even they have become young adults, without which they fear that their children's life prospects may become worrying. Two parents expressed their opinions:

“My mother's time wasn't like how it is now. You now give him a flat or something. Perhaps later when my son needs to buy a flat, I would need to sell mine, yet still not enough for the first down payment. In such case, how do I help him?" (Younger Generation, Group 1, Father A)

"Actually, I do really feel I want to pay the first down payment for my children. I would not pay the monthly instalment, because it would be their responsibility to do so. However, the first down payment is already a huge sum. How do they save so much money? I don't think they have the ability to do so. How much time is needed to save the first down payment?" (Younger Generation, Group 3, Mother D)

In addition to the economic environment, many participants have considered that the decline of the average household size in Hong Kong also contributes to the intergenerational changes in parenting. Nowadays, a typical family usually consists of one or two children. Due to their comparative scarcity, children have become more and more precious and important in the eyes of parents. The image of the precious child hence prevails, and new social norms and 
cultural expectations for giving good care and investing more in child development thus emerged. The following two quotations are good illustrations:

"There goes playgroup for babies. This kind of thing has only become popular in the recent eight or ten years. You see, at a glance, the existence of this is for someone to make money. Nevertheless, many people buy in the idea. True, because we actually are forming a small family with only one or two children... Unlike how my mum and dad who had seven to eight siblings as my grandmother gave birth to seven or eight kids...they could already achieve what playgroups do in their daily interactions." (Younger Generation, Group 1, Father B)

"Family structure nowadays is different from before. Because of the single child situation, children's issues are seen as big problems that need to be fixed as soon as possible. Parents think they have an overall responsibility to deal with the problems." (Social Worker B)

Concomitant to the discourse of the precious child is the focus of Hong Kong parents in enhancing the academic performance of their children. In Hong Kong, the education system is well-known for inducing a lot of stress for children as well as for parents, absorbing much of their energy and constituting a great concern for many parents. The following narratives illustrate how parents face severe pressures to help their children succeed in this education system:

"But her teacher in this school would think, "with your child's talent, she has to be the first or second, but why can she not be the first or second?' So I dare not ask the teacher not to give her so much stress and let her be. I can't say this." (Younger Generation, Group 2, Mother C)

"Parents nowadays would cultivate, explore, and develop children's potential. This might be related to the change in our education system. Parents need to fulfill school's requirement too, and to strive to get children in prestigious schools." (Teacher B)

Besides the societal and familial changes, the professionalization of parent education has also considerably altered the landscape and cultural understanding of parenting. Compared to parenting nowadays, the older generations of parents remarked that professional intervention in parenting was limited in the past, thus they needed to find their own ways to cope with childrearing difficulties. An older parent has recounted her experience in solving childrearing problems by her own effort, while a younger parent has highlighted the difference between the past and the present with regard to learning in parenting as follows:

“As for me, I didn't learn any special attitude in parenting. After I gave birth to my child, I needed to teach him. As he reached different ages and came across different problems, I would then try to solve them. When I didn't know what to do, I would read books or ask someone. In those days... we would think about how to solve the problems only when they appeared. We learnt through our experience, not through a particular curriculum." (Older Generation, Group 1, Mother B)

"When we were young, we were more like nurtured by nature. We wouldn't care what would cause problems to our health, nor would parents care about you very much...Parents probably did not think much about the children but would count on the school... Nowadays, we got to know a lot of different methods to raise children, so we would choose [different childrearing methods]." (Younger Generation, Group 3, Mother B)

Some social workers and parent work practitioners in this study also commented that many existing parent education programs, which are premised on objectivist science and instrumental rationality, have constructed the deficit parent discourse and highlighted the need of parents to learn new knowledge and skills from experts. This may inadvertently heighten their senses of parental anxiety and guilt. Two social workers said:

"Perhaps the social service sector hasn't been sensitive enough to parenting issues. During intervention, 'skills' tend to be overly underlined, which transmitted the stress to parents, and so maybe we also have participated in constructing this social discourse. If parents failed to master the 'so-called knowledge', they would feel they haven't done well enough." (Social Worker B)

"In the recent 10 to 20 years, we have stressed a lot on scientific knowledge and professionalism. We want things to be 'evidence-based' and set up different formulas for people to follow, and emphasize that it is necessary to be so structured as human beings. For example, nowadays if parents are not able to breastfeed, they would feel this is a serious problem. They might even feel so guilty that they would cry." (Social Worker E)

\section{Parents' Responses to the Proliferation of Parenting Experts and Advices}

Whereas many parents highlighted in their narratives the importance of professional knowledge and childrearing advices in parenting, the findings of this study also indicate that they are not just passive containers of professional influences. Rather, they are lively agents who actively draw on their biographical experience and continuous self-reflections to negotiate parenting choices among a diversity of advices in child nurturing and development (To, et al., 2018). First, some parents described themselves as holding a suspicious and cautious stance in digesting the professional knowledge and advices. Two parents had the following remarks:

"I always say, 'it is easy to say'. You [experts] quote sources to support what you said as like this and that. Actually, I feel much information today is rather artificial and is only for commercialization. You see, many people are learning phonics and there are many ways to learn English now. That's easy to earn money, right? Really easy. Now every child needs to learn many things, so how do you filter those information? To me, that's difficult." (Younger Generation, Group 1, Father B)

"Well, about how to filter information, I think there's actually no right or wrong in itself. There is no definite answer. I believe each method has its own statistics to support the belief and that's why there exists such a theory. However, how you choose a suitable belief would depend on the values of yours and your family's and also your children's personality." (Younger Generation, Group 1, Mother C)

Besides based on their own reflections and judgement, some parents are also cognizant of the fact that every child is unique and thus there is no standard method for nurturing a child. Rather, as parents, they should develop the qualities of 
acceptance, patience, and flexibility in nurturing their children. The reflections of these parents are illustrated in the following narratives:

"But I would think... if the advice given by somebody didn't work, then try another way. You know, every child is unique. Because what said by different experts are found to work on different children or parents. So we...Well, I think people in this generation have their own ideas. I would choose by myself." (Younger Generation, Group 3, Mother A)

"That is, [you need to] apply [the knowledge] in your situation. Like the expert whom we have listened to [her talk] that she has the patience towards her children. [She said] you've got to wait for the flowers to bloom, which is unlike how we grab the child tightly. Things like this are helpful to me. I learn some from here, some from there, and then adjust in my childrearing." (Younger Generation, Group 2, Mother A)

\section{DISCUSSION, IMPLICATIONS, AND FUTURE RESEARCH DIRECTIONS}

The findings of this study indicate that parents' and parent work practitioners' reflections on the changing experiences and circumstances of parenting are inextricably related to their lived experiences born out of interactions with the socioeconomic and cultural contexts and dominant social discourses [3]. Their narratives echo the extant studies discussed in literature review by showing that central to the generational shifts of parental beliefs and practices in recent decades in Hong Kong is the drastic expansion of parental responsibilities brought about by the risk-saturated and professional-led discourses which emphasize that parents' efforts are deterministic to the future life chances and developmental outcomes of their children and that parents are responsible for managing the many risks and perils in their children's lives [17]. This conception of parental responsibility encourages parents to see themselves in a technical, functional role, and envision parenting from the perspective of instrumental rationality, which prioritizes doing to being and promotes an interventionist approach focusing solely on the future development of their children [10], [18]. This may in turn limit the vision of parents, impoverished the meanings of parenting, and further undermined the ethical significance of what it means to be a parent of one's own children [18]. That an elevated sense of parental responsibility may also promote intrusive, anxious parental behaviors and in turn bring about both negative parenting and children outcomes [19].

Whereas the participants described vividly how their sense of parental responsibility was being affected by the tremendous demands placed on parenting their children and the many risks and competitions they perceived in their socio-cultural milieu, our findings are different from previous literature by providing a more nuanced picture of the experience and reflections of parents. Although many parents portrayed the socio-cultural environment as an external reality where one could hardly do anything but to submit to its power, some parents highlighted the importance of choice and personal growth as parents, and learn to trust themselves and their children rather than blindly following the professional advice and external trends. As argued by Ferguson [20], "Constructing the self in a post-traditional order is a reflexive project in the sense that critical reflection and incoming information are constantly used by people to constitute and (re)negotiate their identities" (p. 45). Applying this notion of the reflexive self to parenting, our findings indicate that whereas many parents had held a high regard for professional knowledge in parenting, they also reported a gap between such knowledge and their real-life experience and the particular circumstances of their families and children. Therefore, some participants underlined the importance of committing themselves to persistent introspection, negotiating their parental choices and dilemmas in the prevailing intensive parenting culture, and choosing their own actions [21]. In order words, their level of human agency should not be underestimated.

Another interesting finding of the study is the discrepancy between the perception of parents and that of parent work practitioners towards the proliferation of parenting experts and advices. While many parent work practitioners in this study pointed out the adverse influences of the professional-led scientific parenting and childrearing discourses on intensifying parental anxiety and bringing about an intensive approach to parenting, many parents emphasized that those professional advices might at times be helpful for them and thus they should not be discarded altogether. This finding is consistent with the findings of an action research undertaken by Lam and Kwong [14], which indicate that participants of parent education do not regard being taught by professionals as a loss of agency and control. They asked (p. 189), “Are we professionals 'imposing' our ideology of empowerment on parents who in fact cherish expert knowledge? How can parent education be empowering despite this "empowerment paradox"'? From out data it is evident that parents' sense of self can be weakened and jeopardized through their encounter with parenting experts, but at the same time they can also make constructive use of those encounters to reflect on their parental values and beliefs. Therefore, how to strengthen the parental identity and rebuild their parental confidence in parenting intervention is a more significant task instead of discarding all professional input.

By describing in details the changing experiences and circumstance of parenting in Hong Kong, this study explicates the contextualized struggles of parents in making corresponding parenting responses in today's intensive parenting culture. In face of the huge influences of the external conditions of risk society and the existence of various discourses on the experiences and circumstances of parenting, social work and parent intervention practices should help parents fully understand parental anxiety and learn from it, as well as identify the meanings and growth momentums embedded in the changing experiences of parenting [8]. In particular, it should focus on supporting parents to reflect on and construct meanings that transform their life experiences and help them navigate the uncertainties that being a parent may involve, and in turn enable them to make corresponding childrearing responses in line with their values and life experiences.

As we have seen in the narratives of this study, many parents resorted to an individualized approach to parenting 
and held themselves solely responsible for the life outcomes of their children. Such an individualized approach to parenting has its discursive and structural roots in the risk discourses, neoliberal ideologies, and various government policies [7]. In order to lessen the anxiety that this approach has induced on parents, childrearing should be re-conceptualized from a private issue to a community responsibility because childrearing does not exist in a vacuum but is largely influenced and shaped by its specific socio-economic-cultural contexts [11]. The community and the larger ecological context including schools, social service agencies, mass media, the corporate community, etc., all have a part to play in raising the next generation and enhancing the qualities of life and functioning of families [11].

While different generations of parents and practitioners working in different settings were recruited to capture the variations in their perceptions and experiences in this study, given the small sample size, it is still questionable whether other parents and practitioners share the same or similar perceptions and experiences. Moreover, it is questionable whether the research findings are 'valid' representations of reality [22], thus multiple methods of data collection (such as field observations and surveys) should be conducted for data triangulation.

\section{CONCLUSION}

In conclusion, the findings of this study reveal that on the one hand, both older and younger generations of parents and parent work practitioners described parenting in today's Hong Kong society as a risky venture fraught with boundless duties, uncertainties, and challenges, which drives parents towards intensive parenting. On the other hand, some parents thought that while they could not insulate themselves from the current parenting culture, they knew how to figure out their own parental directions and negotiate parental choices and dilemmas among different childrearing options. This paper can thus fill the research gap by generating a comprehensive understanding of the intergenerational changes in childrearing and child development among Hong Kong Chinese parents from the perspective of risk society. It can also shed light on the formulation of education and service strategies for the new generation of parents.

\section{REFERENCES}

[1] F. Shirani, K. Henwood, and C. Coltart, "Meeting the challenges of intensive parenting culture: Gender, risk management, and the moral parent," Sociology, vol. 46, no. 1, pp. 25-40, February 2012.

[2] P. N. Stearns, Anxious Parents: A History of Modern Childrearing in America, New York, NY: New York University Press, 2003.

[3] S. M. To, Y. Y. So, S. M. Iu Kan, K. W. Tsoi, and T. S. Chan, "Supporting parents in late modernity through parent education: A mixed methods study in Hong Kong," J Soc. Work, vol. 18, no. 2, pp. 164-184, March 2018.

[4] U. Beck, Risk Society: Towards a New Modernity. London, U.K. Sage, 1992.

[5] Giddens, Modernity and Self-identity: Self and Society in the Late Modern Age, Stanford, CA: Stanford University Press, 1991.

[6] M. K. Nelson, Parenting Out of Control: Anxious Parents in Uncertain Times, New York, NY: New York University Press, 2010.

[7] R. K. H. Chan, "Risk, individualization and family: Managing the family in Hong Kong," Crit. Soc. Policy, vol. 2, no. 3, pp. 354-367, November 2009.

[8] S. M. To and T. S. Chan, "Searching for the meaning of parenthood: An existential approach to parent education in the Hong

Kong-Chinese context," Int. Soc. Work, vol. 56, no. 4, pp. 467-481, July 2013.

[9] J. L. C. Ma, K. Lai, and S. H. Pun, "Parenting distress and parental investment of Hong Kong Chinese parents with a child having an emotional or behavioral problems," Child Fam. Soc. Work, vol. 7, no. 2, pp. 99-106, May 2002.

[10] T. T. F. Leung and C. M. Lam, "The warrants of parenting: Emotionality and reflexivity in economically disadvantaged families," Int. Soc. Work, vol. 23, no. 3, pp. 353-367, September 2009.

[11] M. Lam, "Parent education: Revision and vision," Asian Journal of Counselling, vol. 10, no. 2, pp. 147-168, 2003.

[12] S. K. Cheung, "Parent education programmes in Hong Kong: Are they effective?" Hong Kong J. Soc. Work, vol. 35, no. 1-2, pp. 85-96, 2001

[13] Leung, M. R. Sanders, S. Leung, R. Mak, and J. Lau, “An outcome evaluation of the implementation of the triple p-positive parenting program in Hong Kong," Fam. Process, vol. 42, no. 4, pp. 531-544, December 2003.

[14] M. Lam and W. M. Kwong, "The "paradox of empowerment" in parent education: A reflexive examination of parents pedagogical expectations," Fam. Relat., vol. 61, pp. 65-74, February 2012.

[15] C. M. Lam and W. M. Kwong, "Powerful parent educators and powerless parents: The 'empowerment paradox' in parent education," J. Soc. Work, vol. 14, no. 2, pp. 183-195, March 2014.

[16] S. M. To, "Development and validation of a quantitative measure for the Chinese sources of parental meaning," J. Child Fam. Stud, vol. 24, no. 12 , pp. 3580-3594, December 2015.

[17] Furedi, Paranoid Parenting: Why Ignoring the Experts May Be Best for Your Child, Chicago, IL: Chicago Review Press, 2002.

[18] J. Suissa, "Untangling the mother knot: Some thoughts on parents, children and philosophers of education," Ethics and Education, vol. 1, no. 1 , pp. 65-77, 2006.

[19] C. Segrin, A. Woszidlo, M. Givertz, and N. Montgomery, "Parent and child traits associated with overparenting," J. Soc. Clin. Psychol, vol 32, no. 6, pp. 569-595, June 2013.

[20] H. Ferguson, "Social work, individualization and life politics," $B r . J$. Soc. Work, vol. 31, no. 1, pp. 41-55, February 2001.

[21] H. D’Cruz, P. Gillingham, and S. Melendez, "Reflexivity, its meanings and relevance for social work: A critical review of the literature," Br. J. Soc. Work, vol. 37, no. 1, pp. 73-90, January 2007.

[22] D. K. Padgett, Qualitative Methods in Social Work Research, 2nd ed. Thousand Oaks, CA: Sage, 2008.
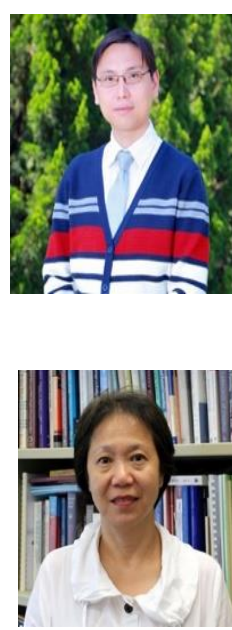

articles.

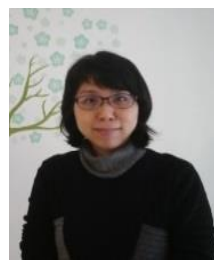

Siu-Ming To is an associate professor of the Department of Social Work of the Chinese University of Hong Kong. He obtained the doctor of philosophy (social welfare) at the Chinese University of Hong Kong in 2006. His research interests are in the areas of life and career development of young people and disadvantaged youth, parenting and parent education, youth empowerment, and program evaluation. He has published more than 40 refereed journal articles.

Ching-man Lam is a professor at the Department of Social Work of the Chinese University of Hong Kong. She obtained the doctor of philosophy at the Wilfrid Laurier University, Canada in 2000.

Her research interests are in the areas of parenting and parent education, marital and family therapy, family and parent empowerment, adolescence and family studies, and social work \& field education. She has published more than 70 refereed journal

Yuk-yan So is a lecturer of the Department of Social Work of the Chinese University of Hong Kong. She received her $\mathrm{PhD}$ in social welfare from the Chinese University of Hong Kong in 2016. Her research interests are in the areas of parenthood and parent education, life and career development, personal growth, and social group work. 